\title{
VPA: Virtual Personal Assistant
}

\author{
Sumitkumar Sarda \\ Department of Information \\ Technology, \\ NBN Sinhgad School of \\ Engineering, \\ Ambegaon, Pune, \\ Maharashtra
}

\author{
Nikita Saibewar \\ Department of Information \\ Technology, \\ NBN Sinhgad School of \\ Engineering, \\ Ambegaon, Pune, Maharashtra
}

\author{
Yash Shah \\ Department of Information \\ Technology, \\ NBN Sinhgad School of \\ Engineering, \\ Ambegaon, Pune, \\ Maharashtra
}

\author{
Monika Das \\ Department of Information \\ Technology \\ NBN Sinhgad School of \\ Engineering, \\ Ambegaon, Pune, \\ Maharashtra
}

\author{
Shivprasad Patil \\ Department of Information \\ Technology, \\ NBN Sinhgad School of \\ Engineering, \\ Ambegaon, Pune, Maharashtra
}

\begin{abstract}
This paper describes the approach to develop a personal assistant that reduces the utilization of input devices like keyboard and mouse on our PC (Personal Computer). Giving commands via speech makes it user friendly. This paper describes the representation model, along with the implementation of this system. Details about the addition of new commands and additional feature of remote access that makes it different from others are also mentioned.
\end{abstract}

\section{Keywords}

Remote access, PC (Personal Computer), Command, Assistant.

\section{INTRODUCTION}

The virtual personal assistant is nothing but an implementation of assistance virtually on the user's PC (Personal Computer). The software can be used via voice, keyboard input and also using internet as the remote access.

There are some predefined commands in the system and user can also add new commands. System can notify the user about new emails, news reports, weather, etc. User can set alarms, reminders, etc. System tasks like shutdown, lock system, sleep etc. are also executed on command. File writing can also be done using this system. The system has a Graphical User Interface which is able to take inputs and give outputs. There are various separate modules for each task like time, alarm, search etc. The Graphical User Interface (GUI) calls these modules for the given commands and so on. Same tasks can be achieved using this software.

The system also has the feature of interacting with the user, which facilitates user and show its artificial intelligence. Also, it has functionalities like defining words, searching documents, files and folders to help user in most possible ways. There are several modules for each task like alarm, search, time etc. These predefined modules are called by the interface when specified in commands.
The system can perform almost all the tasks that require the use of keyboard and/or mouse which is the main goal of the system that will facilitate the disabled people.

Now the features that make this application better are remote access and addition of new commands to the system at user's choice. Using assistant from anywhere is the newest feature as compared to other assistants (like Cortana, Siri). As we can use the assistant by remote access, it removes the limitation of location. We can give commands from any location and get the things done easily. Also user will be able to add new commands which will make the assistant better and user friendly.

\section{PAST WORK}

In addition to prior work focusing on the design of Sirius, an open end-to-end IPA web-service application that accepts queries in the form of images and voice, and responds with natural language. We then use this work to develop the additional feature of remote access [1]. Focus on natural language understanding and dialog management aspects, as author believe that these parts of the technology pipeline require the biggest amount of augmentation [2].

Prior work also describes a multilingual ASR engine embedded on PDA which can support different languages such as English, Mandarin, and Taiwanese. It is based on a one-stage searching strategy and a unified three-layer framework [3].

\section{SYSTEM DESIGN}

The architecture [Fig.1] characterizes the system. User gives text or voice input. Voice commands are converted to text through Google speech API. Text input is simply stored in the database for further process. It is recognised and matched with the commands available in the database. Once the command is found, its respective task is executed as voice, text or through user interface as output. It can also be in the form of application execution. 


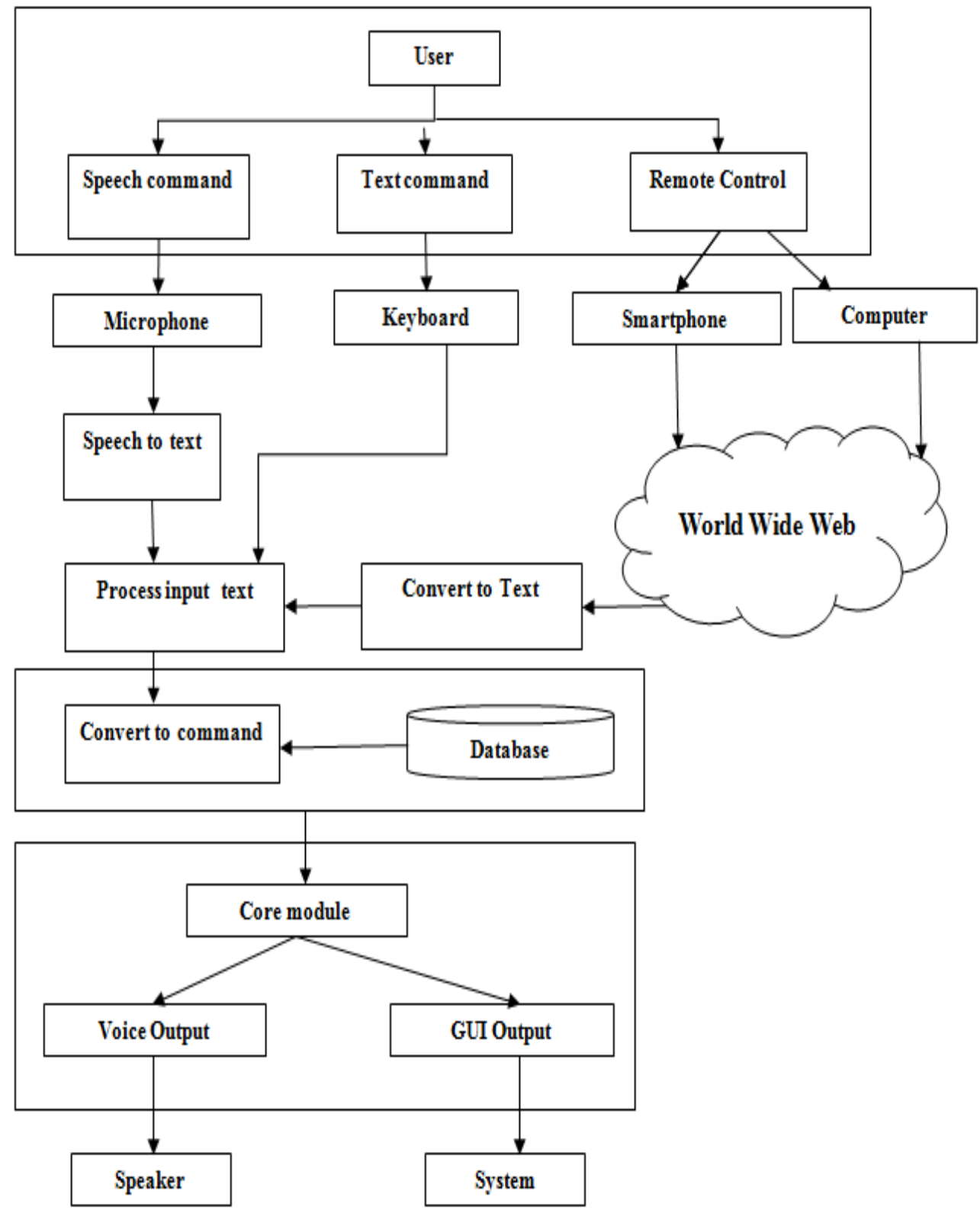

Fig 1: System architecture

\section{MATHEMATICAL} REPRESENTATION

\section{Input Set}

The personal assistant takes voice input, text input and text input through remote signal. So the set of inputs will be,

$I_{1}=\{$ predefined command, fixed pattern sentential command, random sentences as command $\}$

$I_{2}=\{$ voice, text, remote text $\}$

$$
\text { Thus, } \mathrm{I}=\mathrm{I}_{1} \mathrm{U} \mathrm{I}_{2}
$$

Input $\mathrm{I}=\{$ all sentences in English via speech, all sentences in English as text, remote text input $\}$

\section{Output Set}

The outputs for the desired inputs are response determined by the system according to the input given and the database containing all the necessary inputs and their respective outputs.

$$
\begin{aligned}
& \mathrm{O}_{1}=\{\text { voice, display, text }\} \\
& \mathrm{O}_{2}=\{\text { GUI, application response }\}
\end{aligned}
$$

$$
\text { Thus, } \mathrm{O}=\mathrm{O}_{1} \mathrm{U} \mathrm{O}_{2}
$$

Output $\mathrm{O}=\{$ Response for corresponding voice input, Response for corresponding input via GUI, application response \}

There is a one to one relation between input and output. For single input there is only one output. 


\section{Functions}

Following are the operations performed on the input in the system:

\section{Recognize()}

This operation basically gets the input from the user. For text, the input is saved directly into the database while in case of remote signal, the signal is directly converted to the command. For speech input, it converts the voice into text and saves it.

\section{Extract()}

This operation analyses the input string saved and system gets an idea about which command is expected to be executed for the respective input.

\section{Search()}

This operation searches the local database for the response of the command extracted by the previous operation.

\section{Response()}

This operation gives the output that we see on the screen or through speech for the given command.

\section{IMPEMENTATION}

Following is the list of features implemented:

1. Access specific file

2. News alerts

3. Sets alarm

4. Displays date and time

5. Open applications

6. Locks the workstation

7. Power-off the system

8. Hibernates the system

9. Sleep your machine

10. Play typical song or video

11. Weather forecast

12. Know definition of words

13. Search information on Google or Wikipedia

Let's see the implementation of above tasks-

\section{Weather Forecast}

For weather reports, we have used an API called Wunderground. By registering as a developer on the wunderground web site we get an API-key, which is used in the request that the executable sends to the API. The executable gets the weather information in xml format.

http://api.wunderground.com/api/"+wunderground_ key + "/conditions/q/autoip.xml

This xml file detects your location and gives the weather information of your location only.

If we want to get the information of specific city or place then we can use following link,

http://api.wunderground.com/api/"+wunderground_key +

"/conditions/q/" + city_name + ".xml"

The xml from API contains information like-

Place

Observation time

Type of date like weather of forecast etc.

Temperature

Wind

Pressure

Dew point
Visibility $\mathrm{km}$

Latitude Longitude and many more.

\section{Locking, Hibernation, Shutting Down and Rebooting the System}

For these different system calls are used directly from the application these calls are given and the calls are as follows,

\author{
Shut Down \\ shutdown -s -t 10
}

Lockdown

Rundl132.exe User32.dll

\section{Hibernate}

Rundl132.exePowrProf.dll, SetSuspendState

\section{Remote Input}

This is the main feature of this system. We can give input to the assistant by using remote module. Input to the assistant is through internet and LAN or Wi- Fi. For LAN or Wi-Fi, we have created a local Apache web server which contains a webpage which makes the user login into the portal. As the application on user's computer starts, another module is executed in background which sends requests to MySQL databases and check if some new row is inserted. If a new row is detected that command is executed accordingly.

Following query is executed to get the latest command-

SELECT * FROM ‘command1` WHERE ‘id`=" + remote_id + " order by `srno` desc LIMIT 0,1

The remote_id is given on every setup on client PC. It is stored on the user as well as website MySQL databases.

\section{Adding New Commands}

We have used an Ms-Access database to store custom commands as well as some predefined commands. It contains regular expressions with which the input from user are matched and the executable file with arguments given in database only are executed. To add command, user fills the fields like user input, speech response etc. then click on one of the following buttons open a file, open a folder, open a web page and custom speech response. On submitting information required, a simple insert query is performed on Ms- Access database which store the relevant information so that the user expected response is obtained for the given speech input.

\section{Play Typical Songs and Video}

The .NET has a function called Directory.GetFiles (folder_path, filename_pattern); using this function we search all the folders and drives recursively. For videos, we use different extensions like mp4, avi, wmv, flv, mkv, mpg etc. and for songs we search the input song string from user with as, Directory.GetFiles (folder, "*" + name + "*.exe").

Once the file matching with the pattern is found, that file is executed and the respective video or songs starts. This process continues until all the drives and folders are not searched.

\section{RESULTS}

When the application is executed it starts remote module in background. When it gets the command it first tries to match the input with the commands stored in the Ms-Access database if matched it executes the command accordingly. If not then it check the command for some hardcoded flows of matched then the relevant output is given to user. 


\section{CONCLUSION}

Though a number of assistants are available in the market for smartphones, personal assistants for PCs (Windows) are not that popular in our country. An additional feature of remote access eradicates the limitation of area within which the system can be accessed. User can now handle PC (Windows) from mobile or other system through a website. This paper describes the approach that is used to develop a personal assistant. All the technical and implementation details are described to understand the system better. Most of the basic features needed regularly are implemented though a number of additional features can be added. Thus we have developed a personal assistant such that all most of the user tasks are now implemented at the users command easily and quickly.

\section{REFERENCES}

[1] Johann Hauswald, Michael A. Laurenzano, Yunqi Zhang, Cheng Li ,Austin Rovinski, Arjun Khurana, Ronald G. Dreslinski, Trevor Mudge, Vinicius Petrucci1, Lingjia Tang, Jason Mars, "Sirius: An Open End-to-End Voice and Vision Personal Assistant and Its Implications for Future Warehouse Scale Computers",ASPLOS'15, March14-18,2015,Istanbul,Turkey.

[2] P. Milhorat, S. Schl"ogl, G. Chollet, J. Boudy, A Esposito and G. Pelosi," Building The Next Generation
Of Personal Digital Assistants",1st International Conference on Advanced Technologies for Signal and Image Processing - ATSIP'2014 March 17-19, 2014, Sousse, Tunisia

[3] Hong-wen Sie 1, Dau-Cheng Lyu 1, Zhong-Ing Liou 1, Ren-Yuan Lyu 1,2,Yuang-Chin Chiang3 1Dept. of Electrical Engineering, Chang Gung University, Taoyuan," A Multilingual Automatic Speech Recognition (ASR) Engine Embedded on Personal Digital Assistant (PDA)" Cellular Neural Networks and Their Applications, 2005 9th International Workshop

[4] Kaoruko Ohtani, Takahito Niwa,Naohiro Ishii, Nahoko Shirahama, Ippei Torii," Voice Output Communication Aid Application for Personal Digital Assistant for Autistic Children ",2012 IEEE/ACIS 11th International Conference on Computer and Information Science

[5] Yingcbun Yang, Zhaohui Wu, Zhiyou Ma," Real time USER AUTHENTICATION system for PDA "2004 IEEE International Conference on Systems, Man and Cybernetics

[6] Jan Vossaert, Vincent Naessens, Laurens Lemaire "Secure Remote Access to an Industrial Generator with Mobile Devices" World Congress on Industrial Control Systems Security (WCICSS-2015) 